\section{Single-stranded DNA phages}

\author{
Mary R. Lunt
}

The Single-Stranded DNA Phages. Edited by D.T. Denhardt, D. Dressler and D.S. Ray. Pp.720. (Cold Spring Harbor Laboratory: Cold Spring Harbor, New York, 1979.) \$49.

IT is now twenty years since Sinsheimer wrote that finding a single-stranded DNA molecule was "like encountering a unicorn in the ruminant section of the zoo"'. Since then studies on the single-stranded DNA phages have not only produced information relevant to fundamental biological processes but have contributed much to the development of methods now routinely applied in protein and nucleic acid biochemistry.

This book arose from an international meeting held in August 1977, but includes references to papers and unpublished work up to 1978. The articles are comprehensive and well-written reviews of all aspects of the biology of the single-stranded DNA phages. They include discussions of the structural and regulatory roles of the protein-nucleic acid interactions involved, thus providing interest well beyond the specialist areas considered.

The papers mainly refer to the isometric phages $\phi X 174, S 13$ and G4, and to the male-specific filamentous phages $\mathrm{fl}$, fd and M13. They all use Escherichia coli as host and have virions which contain singlestranded, covalently closed, circular DNA of molecular weight about $2 \times 10^{6}$ and coding for nine genes. Besides being the first virus shown to contain such DNA, $\phi \mathrm{X}$ provided the first example of overlapping genes, that is, regions in which the DNA codes for more than one protein, the RNA transcripts being read in different phases. There are articles giving the complete nucleotide sequences of $\phi \mathrm{X}, \mathrm{G} 4$ and $\mathrm{fd}$ DNAs, and discussions of the gene assignments and gene functions. The filamentous phages do not have overlapping genes, and can package variable amounts of DNA, as described in the use of $\mathrm{M} 13$ as a cloning vector.

The chapters on DNA synthesis review the many elegant experiments on the structures of the replicating forms, and their reproduction in vitro. The general significance of these studies is apparent in their analysis of the probable roles of host proteins in bacterial DNA synthesis, and also in their revelation of the importance of DNA secondary structure and its modification by enzymes such as DNA gyrase, helicases and the $E$. coli rep protein.

The analysis of transcriptional control of protein synthesis by these phages has revealed their ability to make a variety of polycistronic RNAs in which sequences coding for proteins made in large amounts appear in more than one RNA species. There are discussions of the structures recognised by RNA polymerase at initiation and termination sites, now made possible by the availability of DNA sequence data.

Increasing attention has recently been given to the formation of intact virions. The isolation of $\phi X$ precursor particles is described and the protein-DNA interactions are discussed. The intriguing production of filamentous phage particles without cell lysis now seems to present an example of the 'signal' mechanism, as nascent coat protein molecules are probably embedded in the cell membrane by way of a leader sequence, the final protein product exchanging with the intracellular DNA binding protein as the

\section{Vitamin D metabolism and function}

\section{R.H. Wasserman}

Vitamin D. By H.F. DeLuca. Pp.80. (Springer: Berlin, New York and Heidelberg, 1979.) DM29; \$15.

VITAMIN D has long been appreciated as the primary factor required for the prevention and cure of rickets and osteomalacia in man and animals. This factor, acquired exogenously or synthesised endogenously by the skin upon exposure to UV light, undergoes biochemical transformations to yield various metabolites, including those hydroxylated in the 25 position [25(OH)D], in the 1 and 25 positions $\left[\mathrm{e}, 25(\mathrm{OH})_{2} \mathrm{D}\right]$ or in the 24 and 25 positions $\left[24,25(\mathrm{OH})_{2} \mathrm{D}\right]$. The $1,25(\mathrm{OH}) \mathrm{D}$ has recently become recognised as a bona fide hormone since it affects reactions elsewhere than at the site of synthsis (kidney) and is under feedback control.

The biological effects of vitamin D and its metabolites have been extensively studied in recent years, and a considerable amount of new information and concepts on this aspect have also become available recently. Among these are the protein synthetic theory of vitamin D action, the identification of several proteins induced or modified by steroid repletion, the interaction of metabolites with the intestine, kidney and bone, and with organs not traditionally considered to be targets, such as the parathyroid gland and pancreas, and details on the translocation of calcium and phosphate across epithelial membranes, to mention just a few.

H.F. DeLuca has gathered together and integrated in a short monograph nuch of phage DNA is extruded from the cell.

The chapters on filamentous phage virion structure provide detailed discussions of diffraction and other data which support different models for the spiral arrangement of protein monomers around the central DNA. There are also accounts of the use of single-stranded DNA phages in the analysis of genetic recombination, and of the disruption of virion structure which occurs when $\phi X$ particles adsorb to the host cell.

The book is not for beginners, but it contains much which can be appreciated and enjoyed by advanced undergraduates and those who teach them.

Mary R. Lunt is Lecturer in Biochemistry at the University of Oxford, UK.

the recent advances. Specifically discussed in this volume are historical aspects, the chemistry of precursors and metabolites of vitamin $D$, the biological control of the formation of vitamin $\mathrm{D}$ by nutritional and hormonal variables, the molecular events affected by vitamin $D$ that bear on calcium and phosphate metabolism, and therapeutic experiences with vitamin $D$ and its metabolites.

Of considerable value are the critical analyses by the author of present concepts and of various published studies. Areas in which there is a paucity of information, and where controversy abounds, are frequently noted. However, not all experts in the field will agree with some of the interpretations and viewpoints of the author, perhaps an expected spin-off of a very active research area. For this reason, the reader is urged to consult other recent reviews and monographs on this topic in order to obtain a broader perspective.

The monograph is well written, lists about 400 references, and is reasonably well illustrated. Since the author and his associates around the world have been prolific contributors to vitamin D research, this monograph in a sense represents a cataloguing of their many contributions (about $30 \%$ of the references cited are authored or co-authored by Professor DeLuca).

This monograph should appeal both to the specialist on vitamin $\mathrm{D}$, calcium and phosphorus metabolism, and to the interested non-specialist. It should also prove useful to the clinician, providing an introduction to the chemical and biological behaviour of a class of compounds that are efficacious in some disease states and potentially therapeutic in others.

R. H. Wasserman is a Professor in the Department of Physical Biology and Section of Physiology, New York State College of Veterinary Medicine, Cornell University, Ithaca, New York. 